\title{
Impact of Eastern Congo Ebola Outbreak on Child Health Services Delivery: Decline of Routine Child Immunization and Need for Vaccine Supplementation
}

Nzaji Michel-Kabamba ( $\square$ christopherkabamba64@gmail.com )

University of Lubumbashi and Kamina

Lungoyo Christophe-Luhata

Universite de Lubumbashi

Nlandu Roger Ngatu

Kokusai Iryo Fukushi Daigaku

Charlotte Othepa

Universite de Lubumbashi

Mwimba Bertin-Lora

Universite de Lubumbashi

Mukola Arsene-Kabwaya

Universite de Lubumbashi

Stephane H. Bateyi

Universite de Lubumbashi

Nicole A. Hoff

University of California Berkeley School of Public Health

Ngombe Leon-Kabamba

Universite de Lubumbashi

Basilua Andre Muzembo

Kokusai Iryo Fukushi Daigaku

Koji Wada

Kokusai Iryo Fukushi Daigaku

Shunya Ikeda

Kokusai Iryo Fukushi Daigaku

Elisabeth Mukamba

Universite de Lubumbashi

Mwamba Guillaume-Ngoie

Universite de Lubumbashi

Numbi Oscar-Luboya

Universite de Lubumbashi Faculte de Medecine 


\section{Research article}

Keywords: Democratic Republic of Congo; Ebola virus disease; Child immunization; Outbreak.

Posted Date: December 3rd, 2019

DOI: https://doi.org/10.21203/rs.2.15848/v2

License: (c) (1) This work is licensed under a Creative Commons Attribution 4.0 International License. Read Full License 


\section{Abstract}

Background An Ebola disaster, the deadliest in the history of Ebola in the Democratic Republic of Congo (DRC), is unfolding in North Kivu and Ituri provinces. An interdisciplinary research team investigated the impact of the Ebolavirus disease (EVD) outbreak on vital health services delivery under the coordination of DRC National Vaccination Program, Ministry of Health. We report on the impact of the ongoing Eastern Congo EVD outbreak on routine child immunization in the EVD-affected in North Kivu province, DRC. Methods This was a retrospective longitudinal study consisting in a time-trend analysis that was conducted in 40 public health facilities that organize routine child immunization in four of the 34 'Health Zones' of the North Kivu province, namely Beni, Butembo, Katwa and Mabalako. Data were collected during two distinct 3-month periods: the "pre-Ebola period" (1 May through July 2018) and the "Ebola period" (1 August through October 2018), to compare the vaccination trends. Mean number of vaccine doses administered in pre-Ebola and Ebola periods were compared (unpaired $t$ test). Results Compared to the "pre-Ebola period", overall monthly mean vaccines uptake dropped during the "Ebola period": BCG (157.1 \pm 150.3 vs. $227.5 \pm 138.4$; $p<0.0001$ ), Oral polio (OPV: $505.1 \pm 474.6$ vs. $631.3 \pm 353.9 ; p<0.001$ ), Measles vaccine (147.6 \pm 126.7 vs. $184.7 \pm 102.5 ; p<0.001)$, pneumoccocal vaccine (PCV13: $445.8 \pm$ 395.5 vs. $554.3 \pm 299.8 ; p<0.001)$ and inactivated polio vaccine (IPV: $183.9 \pm 141$ vs. $153.9 \pm 146.6$; $\mathrm{p}$ <0.01). Furthermore, when considering the trend in child immunization in each of the four Health Zones during 'Ebola period', vaccine uptake shrank considerably in most health zones, whereas a complete disruption of vaccine delivery was observed in Mabalako health zone where the epicenter of this outbreak is located. Conclusion The ongoing Ebola outbreak has a negative impact on child health services, routine vaccination in particular. Supplementary immunization coupled with the strengthening of routine child vaccination services should be implemented to address gaps in immunity among children in affected provinces and reduce the risk of other infectious diseases outbreaks. Keywords: Democratic Republic of Congo; Ebola virus disease; Child immunization; Outbreak.

\section{Introduction}

The first Ebolavirus disease (EVD) outbreak first occurred in 1976 in a village near the Ebola river in the Democratic Republic of Congo (DRC, formerly Zaire) [1, 2]. Since then, there have been 10 confirmed EVD outbreaks in DRC and overall 35 epidemics in the world, including the 2014-2016 West African outbreak which ended with more than 28,600 cases and 11,325 deaths [3,4]. The ongoing East-Congo EVD outbreak was declared on 1 August 2018 at Mabalako Health Zone in Beni, North Kivu province [5]. It is the most severe EVD outbreak to occur in DRC. A recent report from the World Health Organization (WHO) shows 3,091 reported cases of whom 2,980 confirmed and 111 probable cases; a total of 2,074 cases died (as of 10 September 2019) [6].

The epidemic has recently crossed the country's border, with confirmed cases from a family in Kasese district, Uganda, that stayed in Mabalako during the funerals of a relative who was a Congolese pastor infected by Ebolavirus [7]. The eastern DRC is a region affected by two decades of armed conflict and extreme gender-based violence (including rapes of women and children) perpetrated by soldiers and 
armed militia members, since the beginning of the Congo war in 1998; and North Kivu is one of the provinces that have been bearing the heaviest burden of this conflict $[8,9]$.

While EVD can have major impacts on mortality and morbidity from infection as seen from the 20142016 West African outbreaks, it can additionally have other health and societal consequences unrelated to infection. Consequences include social instability, poor food reserves, breakdown of healthcare systems and reduced vaccination coverage for children under five. Breakdown of healthcare systems and reduced vaccination coverage were considered the worst consequences during the West African EVD outbreak as nearly all health resources were shifted to the EVD response [10]. Disruptions of local health systems could lead to underreporting of other diseases leading to a second crisis that could negatively impact as many as persons as the original outbreak, if not more [9]. Several studies have described this phenomenon during the West African EVD outbreak, showing the movement of resources to response efforts were responsible for significant disruptions to the provision of routine health care [11-13].

Up-to-date, less information has been available on the impact of the Ebola outbreak on preventive child health services such as routine vaccination, particularly in DRC. In fact, DRC is the country that has experienced the highest number outbreaks in the history of EVD; however, no scientific report on the child vaccination issue has been available in the literature. Maintaining routine immunization activities during an outbreak of a communicable disease such as EDV is vital to prevent other vaccine-preventable diseases and associated morbidity and mortality. It has been projected that due to the disruption in the routine immunization services for $12-18$ months, around $25-75 \%$ reduction in the vaccination rates have been observed at country level since the end of the West-African Ebola outbreak [14].

The present study aimed to examine the impact of the ongoing East-Congo Ebola outbreak on childhood vaccination and assess the trend in vaccination activity across the pre-Ebola and Ebola periods in North Kivu province, DRC.

\section{Methods}

\section{Study design and sites}

A retrospective longitudinal study, consisting in a time-trend analysis, was conducted in 40 public health facilities that organize routine child vaccinations, located in four of the 34 health zones of the North Kivu province, namely Beni, Butembo, Katwa and Mabalako (Fig. 2) [15]. The latter is the name of the Health Zone where Mangina, the epicenter of the ongoing EVD outbreak, is located. North Kivu is one of the EastCongo provinces that are still suffering from a two decade of conflict related atrocities perpetrated by foreign and local armies and militias, resulting in an unprecedented and generalized insecurity, shortage of health care providers and the breakdown of the provincial health system.

\section{Data collection}


Data were from compiled standardized vaccination tally sheets used in health facilities and reported to the Health Zone office on a monthly basis, in conformity with recommendations from the DRC National Vaccination Program. For this study, we used data recorded from May through October 2018, and two periods of 3 months each were considered to compare the trend of vaccine uptake: the "pre-Ebola period" (May-July 2018) and the "Ebola period" (August-October 2018). These periods correspond to the period before and after the official declaration of the outbreak, respectively. Data collection was undertaken simultaneously in all 40 health facilities by experts of the DRC National Vaccination Program, Ministry of Health. They were transcribed in prepared excel files for statistical analyses.

Declared in early August 2018, the ongoing Eastern Congo Ebola outbreak started at Mangina area in Mabalako Health Zone in North Kivu province, then spread within Mabalako. Latter on, new cases were reported in Health Zones located around Mabalako such as Katwa, Beni and Butembo before reaching the Ituri province within the same month. This largest-ever epidemic to occur in DRC is ongoing in a densely-populated region with cross-border population flow with neighboring countries such as Uganda, South Sudan, Rwanda and Burundi. It has been declared a public health emergency of international concern on 17 July 2019.

\section{Outcome variables}

The main outcome variable was the number of vaccination procedures performed during each of the study periods. Besides, other information related to type of health facility and the vaccine types [Bacilli Calmette-Guerin (BCG), Pentavalent vaccine, oral polio vaccine (OPV), Measles vaccine, anti-amaryl vaccine (AAV), inactivated polio vaccine (IPV), Tetanus vaccine, pneumococcal vaccine (PCV13)] was collected.

\section{Data analysis}

In order to reduce the possible influence of seasonal difference in the number of vaccination procedures, comparisons between pre Ebola and Ebola periods were limited to six months. Mean values of the number of vaccine doses administered per month in the pre and ongoing Ebola periods were compared using unpaired $t$ test. All the analyses were performed with the use of SPSS 23 software, and the significance level of difference was set at $p<0.05$ (double-sided).

\section{Results}

Change in vaccine uptake in all four Health Zones (Beni, Butembo, Katwa, Mabalako) before and after the declaration of the ongoing East-Congo Ebola outbreak

Table 1 shows the trend of number of vaccine doses administered monthly in all 40 public health settings of the four selected health zones in North Kivu province, according to vaccine types. It was observed that the number of vaccine doses administered during the pre-Ebola period was significantly higher for all 
vaccine types compared to the Ebola period: $195.3 \pm 280.8$ vs. $157.1 \pm 150.3$ for BCG $(p<0.001)$, $484.9 \pm 298.6$ vs. $444.3 \pm 393.1$ for pentavalent vaccine ( $p<0.001), 549.6 \pm 345.3$ vs. $505.1 \pm 474.6$ for OPV ( $p<0.001$ ), $175.2 \pm 96.0$ vs. $147.6 \pm 126.7$ ( $p<0.001$ ) for VAR, $167.2 \pm 92.3$ vs. $142.4 \pm 124.5$ for VAA, $163.9 \pm 100.0$ vs. $154.0 \pm 146.6$ for IPV, $197.1 \pm 155.8$ for Tetanus vaccine, $475.0 \pm 301.7$ vs. $445.8 \pm 395.5$ for PCV13 $(p<0.01)$, respectively.

\section{Vaccine uptake in each of the Health Zones during the pre-Ebola and Ebola periods}

Figure 2 shows the monthly trends in under-five vaccination by vaccine type administered at healthfacility level during the pre-Ebola and Ebola periods in all four Health Zones. In Beni, a decline of monthly number of vaccine doses was observed in September, a month after the declaration of the outbreak, the four antigens (BCG, OPV, Pentavalent, Measles). In addition, a similar trend was observed for the four other antigens (PCV 13, Tetanus vaccine, IPV, VAA), but not significantly (Fig. 2A, B). On the other hand, though the number of vaccine doses administered in Butembo Health Zone tended to be not negligible for BCG, Pentavalent, VAA, IPV, PCV13 and Tetanus vaccine in August 2018, a sudden drop in routine child immunization was observed for all vaccine types in September and October 2018 (Fig. 2 C, D).

Regarding the status of routine child immunization service in Mabalako Health Zone where the epicenter of the current EVD outbreak is located, a striking drop of vaccine uptake was observed for all antigens (BCG, Pentavalent, OPV, Measles, VAA, IPV, Tetanus and PCV13 vaccines), suggesting the absence of child immunization activity from August to October 2018 in the area (Fig. 2 E, F). Furthermore, in Katwa Health Zone, a relative reduction in vaccines doses administered was observed for BCC and Pentavalent vaccines in October 2018 but not significantly. It remained almost unchanged for OPV, Measles, and Tetanus vaccines. Regarding PCV13, a drop in vaccine uptake was noted in July 2018 (pre-Ebola period). Then, an increase in August 2018 during the Ebola period, which was followed by a sharp decrease in vaccine uptake in September and October 2018 (Ebola period) (Fig. 2 G, H).

\section{Discussion}

This study adds to the growing pool of literature concerning vaccination trends before and during an Ebola outbreak, and is the first from the DRC, a country that has experienced the most EVD outbreaks since its discovery in 1976. There was a significant decrease in the number of vaccine doses administered monthly for all antigens received during the Ebola outbreak (August-October 2018) as compared with the pre Ebola period (May-July 2018). Our results corroborate those found in several other countries affected by EVD $[11,16]$.

Additionally, there was an overall reduction in the number of children vaccinated during the "Ebola period", which is consistent with the notion of reduced health services use secondary to community perception of increased risk of contracting Ebola. This, coupled with the 'no touch' policy in which health workers were ordered not to practise invasive procedures, might have contributed to the reduction in the 
number of vaccines provided, as most vaccines (except polio vaccine) require an injection into either the upper arm or thigh. The priority of Ebola response and control, coupled with constraints in logistical support to ensure vaccine delivery and timely reporting, may also have played a role in the decline in vaccination coverage among health facilities in the areas most affected by Ebola.

Furthermore, rumors surrounding the current Ebola outbreak (some local residents suspect a man-made Ebola outbreak by those military groups that have been killing civilians) - in an area ravaged by two decades of insecurity due to armed conflicts and extreme violence - might have spread the fear among the eastern Congo population $[\mathbf{1 7}, \mathbf{1 8}]$. This fact might have also contributed to reducing vaccines uptake in affected areas.

In regards to child vaccination in the context of EVD outbreak, another study reported that numbers of $B C G$, pentavalent, and measles vaccine doses administered remained relatively stable, with some decreases. Our study reveals a sudden shrink in vaccines uptake in two of the four surveyed Health Zones, whereas a total absence of vaccine uptake (for all vaccine types) was observed in Mabalako Health zone where the epicenter of the EVD epidemic is located, in the three month-period (from August to October 2018) following the declaration of the outbreak.

In the context of the ongoing Ebola outbreak in DRC, a look at data on child immunization activity during a similar period (August-October) in 2017 showed that routine vaccination was implemented regularly in Mabalako Health Zone, with OPV reaching over 1,000 doses delivered (1,416 doses in August, 1,191 in September, and 1,248 in October 2017). Thus, the ongoing eastern Congo EVD outbreak caused large losses in vaccination outputs, leaving young children at significant risk for infection with life-threatening illnesses, as well as potentially putting adults at risk through the breakdown of community-level herd immunity [19].

Epidemics of measles are often an early result of interruptions in the delivery of public health services. Historically, measles outbreaks have followed humanitarian crises, such as war [20], natural disasters and political crises [10]. Measles is one of the most transmissible infections, and immunization rates tend to be lower due, in part, to the age at which measles vaccine must be administered [14].

Based on surveys from health care providers, it was assumed that a $75 \%$ reduction in vaccination rates has been observed after the west African Ebola outbreak, with the projection that the number of children between 9 months and 5 years of age not vaccinated against measles would increase by an average of almost 20000 every month, reaching more than 1 million unvaccinated children by 18 month [21]. Similar reductions in the rate of vaccination would increase the number of children not receiving a pentavalent vaccine, BCG and oral polio vaccine.

The setbacks in vaccination rates observed in Ebola-affected area have the potential to erode the substantial gains in the control of these diseases made in recent decades, and a large population of children susceptible to poliovirus infection could threaten the Global Polio Eradication Initiative, should wild poliovirus be re-introduced. However, childhood vaccine-preventable diseases (VPDs) are an area 
where there is a clear, relatively inexpensive, and one-time intervention that could erase the impact of Ebola related health care disruptions.

Coordinated campaigns across Ebola affected Health Zones should include targeting children who were most likely to have missed one or more critical routine vaccinations to prevent the occurrence of VPDs [22-24] such as Measles during the Ebola epidemic. More importantly, a campaign should target children aged 6 months to 5 years (typical of the age range targeted by follow-up supplementary immunization activities (SIAs), perhaps extending the lower age range and administering childhood vaccines other than measles vaccine to the youngest children. Planning for such an immunization campaign should begin imperatively in order to reduce the risk of disease outbreaks in children population.

Nonetheless, the present study has some limitation. The assessment of vaccination coverage was limited

to the health zone that was the first to be affected and three other Health Zones located quite close to the epicenter. This was based on the assumption that health zones near the epicenter would be the most affected at the early phase of the Ebola outbreak. On the other hand, the reduced number of vaccinations could have been caused by other factors such as underreporting of routine immunization due to EVD outbreak in the area. However, after interviewing the Health Zone managers, neither such an issue nor a shortage in hospital personnel was noted.

\section{Conclusion}

This study showed a marked decrease in the number of vaccine doses administered for all antigens during in the "ongoing Ebola period" in almost all Health Zones that were considered in this study. In particular, vaccination service delivery was disrupted in Mabalako health Zone where the ongoing EVD outbreak is located, suggesting a dysfunction of health services following the declaration of the EVD outbreak.

This research-based evidence on the impact of Ebola outbreak on child immunization in DRC should be considered by Congolese health authorities for better policies to be implemented in case of occurrence of similar emergency events. Findings from this study suggest the need for measures to be taken in order to avoid such disruptions in health services delivery, and for SIAs to reduce the risk of VPD outbreak.

\section{List Of Abbreviations}

- AAV: anti-amaryl vaccine

- BCG: bacilli Calmette-Guerin

- DRC: Democratic Republic of Congo

- EVD: Ebola virus disease

- IPV: inactivated polio vaccine

- OPV: oral polio vaccine 
- PCV: pneumoccocal vacine

- SIA: supplementary immunization activity

- WHO: World Health organization

- VPD: vaccine preventable disease

\section{Declarations}

\section{Ethics approval and consent to participate}

Given that this was an aggregate data review study with anonymized data collected as a part of routine National Vaccination Program services; thus, informed consent was not required. Nonetheless, ethical clearance was obtained from the ethics committee of Lubumbashi Medical College.

\section{Consent for publication}

All authors read the last version of this paper and agreed that it can be published.

\section{Availability of data and material}

The source data related to this work are available at the DRC National Vaccination Program, DRC Ministry of Health, and can be requested to the Vice-director of the program (NMK).

\section{Competing interests}

The authors declare no competing interest related to this study.

\section{Funding}

No external fund was obtained. All activities of this work were supported by the DRC National Vaccination Program.

\section{Authors' contributions}

- NMK, LCL, CO, MGN, NOL, MAK, SHB and MBL designed the study and conducted related fieldwork.

- NRN, MK, KW, LCL, MAK, NLK, NAH and MBL conducted literature review, and participated in data collection and analysis.

- MK, MGN, SI, KW, BAM, NOL, MEM and NRN interpreted the results and drafted the manuscript. All authors read the manuscript and approved its final version; NAH and NRN edited the paper.

\section{Acknowledgements}

The authors thank the managers of Health Zones for their support to this study.

\section{References}


1. Breman JG, Heymann DL, Lloyd G, Mccormick JB, Miatudila M, Murphy FA. Discovery and Description of Ebola Zaire Virus in 1976 and Relevance to the West African Epidemic During 2013 2016. J Infect Dis. 2016; 214(3): 93-101.

2. Ngatu RN, Kayembe NJM, Phillips EK, Okech-Ojony J, Patou-Musumari M, Gaspard-Kibukusa M, Madone-Mandina N, Godefroid-Mayala M, Mutaawe L, Manzengo C, Roger-Wumba D, Nojima S. Epidemiology of ebolavirus disease (EVD) and occupational EVD in health care workers in SubSaharan Africa: Need for strengthened public health J Epidemiol. 2017; 27(10): 455-461.

3. WHO Ebola Response Team. After ebola outbreak in west Africa - unpredictable risks, preventable epidemics. New Engl J Med. 2016; 11: 587-596.

4. Centers for Disease Prevention and Control (CDC). 2014-2016 Ebola outbreak in west Africa. Available from: https://www.cdc.gov/vhf/ebola/history/2014-2016-outbreak/index.html (Accessed 2019 May 6)

5. Claudel KM, Underschultz J, Hawkes MT. Ebola virus epidemic in war-torn eastern DR Congo. The Lancet. 2018; 392(18): 1399-1401.

6. Ebola virus disease - Democratic Republic of Congo. Available from:https://www.who.int/csr/don/12-september-2019-ebola-drc/en/ (Accessed 25 September 2019).

7. Brown C. Rape as a weapon of war in the DemocraticRepublic of Congo. Torture. 2012; 22(1): 24-37.

8. World Health Organization (WHO). Emergencies preparedness, response. Ebola virus diseaseRepublic of Uganda. Available from:https://www.who.int/csr/don/13-june-2019-ebola-uganda/en/

9. Bress J, Kashmwa G, Amisi C, Armas J, McWhorter C, Ruel T, Ammann AJ, Mukwege D, Butler LM. Delivering integrated care after sexual violence in the Democratic Republic of Congo. Lancet Glob Health. 2019; 22: 4(1): e001120

10. Colavita F, Biava M, Castilletti C, Quartu S, Vairo F, Caglioti C, Agrati C, Lalle E, Bordi L, Lanini S, Guanti MD, Miccio R, Ippolito G, Capobianchi MR, Di Caro A; Lazzaro Spallanzani Institute for Research and Health Care Ebola Virus Disease Sierra Leone Study Group.. Measles cases during ebola outbreak, West Africa, 2013-2106. Emerg Infect Dis. 2017; 23 (6): 1035-1037.

11. Camara BS, Delamou A, Diro E, El Ayadi MA, Beavogui AH, Sidibé S, Grovogui FM, Takarinda KC, Kolié D, Sandouno SD, Okumura J, Baldé MD, Van Griensven J, Zachariah R. Influence of the 20142015 Ebola outbreak on the vaccination of children in a rural district of Guinea. Public Health Action. 2014; 1(2): 116-121.

12. Delamou A, Delvaux T, El Ayadi AM, Beavogui AH, Okumura J, Van Damme W, De Brouwere V. Public health impact of the 2014-2015 Ebola outbreak in West Africa: seizing opportunities for the future. BMJ Global Health. 2017: 2(2), e000202.

13. Plucinski MM, Guilavogui T, Sidikiba S, Diakité N, Diakité S, Dioubaté M, Bah I, Hennessee I, Butts JK, Halsey ES, McElroy PD, Kachur SP, Aboulhab J, James R, M. Effect of the Ebola-virus-disease epidemic on malaria case management in Guinea, 2014: a cross-sectional survey of health facilities. Lancet Infect Dis. 2015: 15(9): 1017-1023. 
14. Takahashi S, Metcalf CJE, Ferrari MJ, Moss WJ, Truelove SA, tatem AJ, Grenfell BT, Lessler J. The growing risk from measles and other childhood infections in the wake of Ebola. 2016: 347(6227): 1240-1242.

15. Centers for Diseases Control and Prevention (CDC). 2018 Eastern Democratic Republic of the Congo Ebola outbreak. Available from:https://www.cdc.gov/vhf/ebola/outbreaks/drc/east-drc-map.html (accessed 8 June 2019)

16. Wesseh CS, Najjemba R, Edwards JK, Owiti P, Tweya H, Bhat P. Did the Ebola outbreak disrupt immunisation services? A case study from Liberia. Public Health Action. 2017: 7(1), 82-87.

17. Wagenaar BH, Augusto O, Beste J, toomay SJ, Wickett E, Dunbar N, Bawo L, Wesseh CS. The 20142015 Ebola virus disease outbreak and primary healthcare delivery in Liberia: Time-series analyses for 2010-2016. PLoS Med. 2018; 15(2), 1-26.

18. Trapido J. Ebola: public trust, intermediaries, and rumour in the DR Congo. Lancet Infect Dis 2019; 19(5): 457-458.

19. Vinck P, Pham PN, Bindu KK, Bedford J, Nilles EJ. Institutional trust and misinformation in the response to the 2018-19 Ebola outbreak in North Kivu, DR Congo: a population-based surpvey. Lancet Infect Dis 2019; 19(5): 529-536.

20. Sharara SL, Kanj SS. War and Infectious Diseases: Challenges of the Syrian Civil War. PLoS Pathogens 2014; 10(11): 2-5.

21. Friedrich MJ. Increased Measles Risk Possible in African Countries Affected by Ebola. JAMA. 2015; 313(19): 1897 (2015).

22. Biellik RJ, Orenstein WA. Strengthening routine immunization through measles-rubella elimination. Vaccine. 2018; 36(37): 5645-5650.

23. Fields R, Dabbagh A, Jain M, Sagar KS. Moving forward with strengthening routine immunization delivery as part of measles and rubella elimination activities. Vaccine 2013; 31(2): B115-B121.

24. Centers for Diseases Control and Prevention (CDC). 2018 Eastern Democratic Republic of the Congo Ebola outbreak. Available from: https://www.cdc.gov/vhf/ebola/outbreaks/drc/east-drc-map.html

\section{Tables}

Table 1. Mean number of vaccine doses administered per month during the pre-ebola and ebola periods in all four Health Zones, May -October 2018 


\section{Vaccine type}

BCG (Bacille Calmette-Guerin)

Pentavac (pentavalent vaccine)

OPV (oral polio vaccines)

Measles vaccine

AAV (anti-Amaryl vaccine)

IPV (inactivated polio vaccine)

Tetanus vaccine

PCV 13 (pneumococcal vaccine)
Pre-Ebola period Ebola period

p-value

$($ Mean \pm SD) $\quad($ Mean \pm SD)

$227.5 \pm 138.4 \quad 157.1 \pm 150.3<0.0001$

$484.9 \pm 298.6 \quad 444.3 \pm 393.1<0.01$

$631.3 \pm 353.9 \quad 505.1 \pm 474.6 \quad 0.001$

$184.7 \pm 102.5 \quad 147.6 \pm 126.7<0.001$

$183.3 \pm 104 \quad 142.4 \pm 124.5<0.001$

$183.9 \pm 99.8 \quad 153.9 \pm 146.6<0.01$

$188.9 \pm 141 \quad 152.8 \pm 172.6<0.05$

$554.3 \pm 299.8 \quad 445.8 \pm 395.5<0.001$

\section{Figures}

Fig. 1

Figure 1

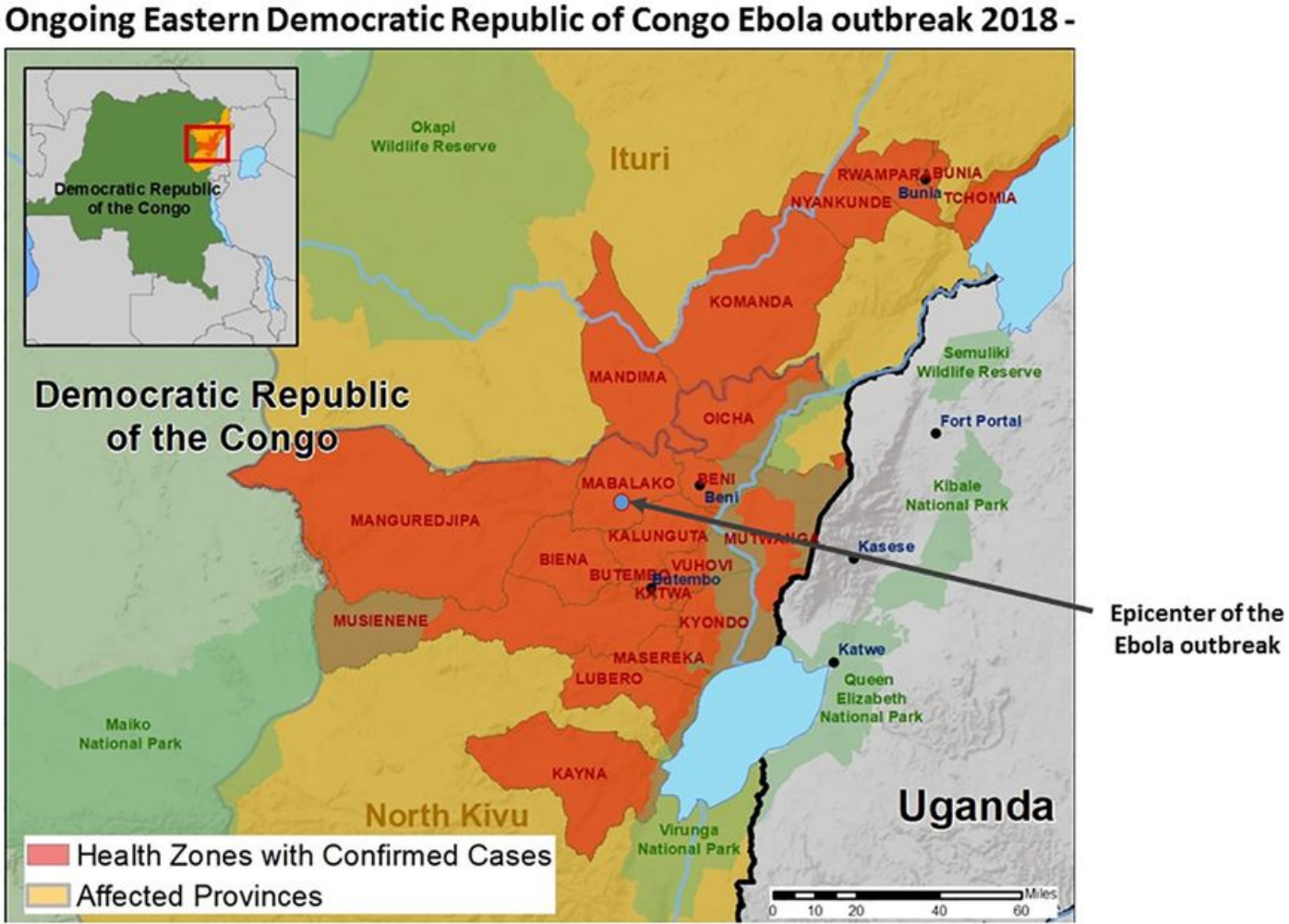


Map of Eastern Congo provinces of North Kivu and Ituri showing Health Zones affected by the ongoing Ebola outbreak, with the epicenter located in Mabalako (CDC ebola map, 2019)15 [Permission for reproduction of this image was obtained from CDC, USA]
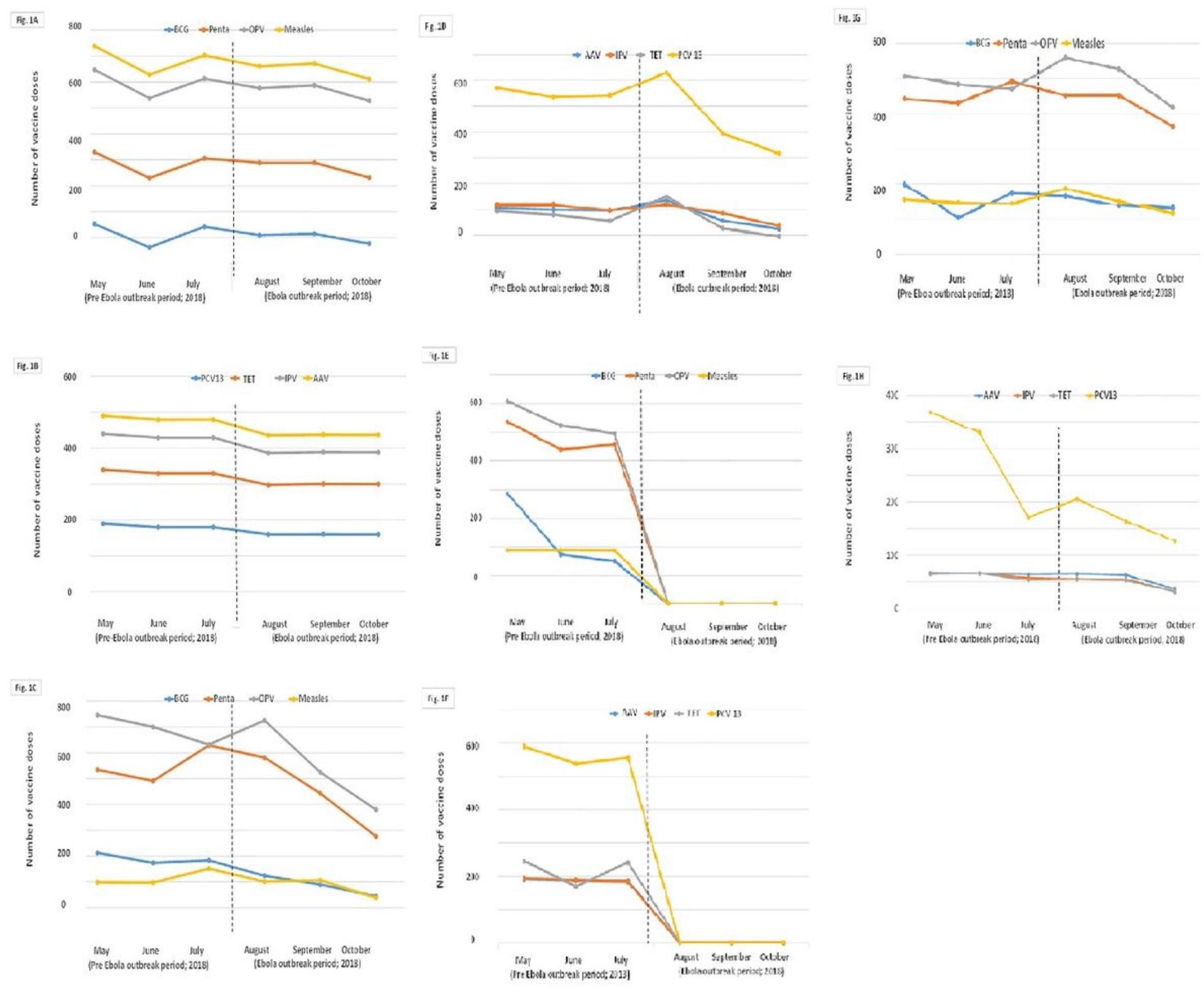

\section{Figure 2}

Trend in the number of vaccine doses administered during routine child immunization services before and after Eastern Congo Ebola outbreak declaration in Beni (A, B), Butembo (C, D), Mabalako (E, F) and Katwa $(G, H)$ Health Zones -Legend: BCG, Baccili Calmette-Guerin; OPV, oral polio vaccine; AAV, antiamaryl vaccine; IPV, inactivated polio vaccine; PCV13, pneumocccal vaccine 13; Penta, Pentavalent vaccine; TET, Tetanus vaccine. The figure shows an overall decline in vaccine uptake in the Ebola period in Beni and Butembo Health Zones as compared with the status of routine vaccination in pre-Ebola period, whereas no routine vaccination was administered during the first 3- month period of the Eastern Congo Ebola outbreak (August-October 2018). 